\title{
Spatial-temporal analysis of malaria and the effect of environmental factors on its incidence in Yongcheng, China, 2006-2010
}

Yan Zhang ${ }^{1,2}$, Qi-Yong Liu ${ }^{1,3,4^{*}}$, Rong-Sheng Luan ${ }^{5}$, Xiao-Bo Liu' ${ }^{1}$ Guang-Chao Zhou ${ }^{6}$, Jing-Yi Jiang ${ }^{6}$, Hong-Sheng $\mathrm{Li}^{6}$ and Zhi-Fang $\mathrm{Li}^{1}$

\begin{abstract}
Background: In 2003, Plasmodium vivax malaria has re-emerged in central eastern China including Yongcheng prefecture, Henan Province, where no case has been reported for eleven years. Our goals were to detect the spacetime distribution pattern of malaria and to determine significant environmental variables contributing to malaria incidence in Yongcheng from 2006 to 2010, thus providing scientific basis for further optimizing current malaria surveillance and control programs.

Methods: This study examined the spatial and temporal heterogeneities in the risk of malaria and the influencing factors on malaria incidence using geographical information system (GIS) and time series analysis. Univariate analysis was conducted to estimate the crude correlations between malaria incidence and environmental variables, such as mosquito abundance and climatic factors. Multivariate analysis was implemented to construct predictive models to explore the principal environmental determinants on malaria epidemic using a Generalized Estimating Equation (GEE) approach.

Results: Annual malaria incidence at town-level decreased from the north to south, and monthly incidence at prefecture-level demonstrated a strong seasonal pattern with a peak from July to November. Yearly malaria incidence had a visual spatial association with yearly average temperature. Moreover, the best-fit temporal model (model 2) $\left(\mathrm{QIC}=16.934, \mathrm{P}<0.001, \mathrm{R}^{2}=0.818\right)$ indicated that significant factors contributing to malaria incidence were maximum temperature at one month lag, average humidity at one month lag, and malaria incidence of the previous month.

Conclusions: Findings supported the effects of environment factors on malaria incidence and indicated that malaria control targets should vary with intensity of malaria incidence, with more public resource allocated to control the source of infections instead of large scale An. sinensis control when malaria incidence was at a low level, which would benefit for optimizing the malaria surveillance project in China and some other countries with unstable or low malaria transmission.
\end{abstract}

Keywords: Malaria, Anopheles, Weather, Geographic information system, Modeling

\footnotetext{
* Correspondence: liuqiyong@icdc.cn

${ }^{1}$ State Key Laboratory for Infectious Diseases Prevention and Control,

National Institute for Communicable Disease Control and Prevention,

Chinese Center for Disease Control and Prevention, Beijing, People's Republic

of China

${ }^{3}$ China CDC Key Laboratory of Surveillance and Early-Warning on Infectious

Disease, Beijing, People's Republic of China

Full list of author information is available at the end of the article
} 


\section{Background}

Malaria is one of the major causes of morbidity and mortality in the world, with less than one million deaths annually reported by WHO [1]. Malaria is a serious global public health problem, and its prevention and control is addressed in the United Nations (UN) Millennium Development Goals (MDG) [2]. Yongcheng prefecture, Henan province is a research center of the 2010-2020 plan for national malaria elimination in China [3], which is an important part of global malaria action plan (GMAP) [4]. In the past, malaria in Yongcheng prefecture were severe, with incidence as high as 3.34 per 100,000 population in 1970 . Malaria incidence was drastically reduced after national comprehensive interventions, which included case management and Anopheles elimination. In 2003, malaria re-emerged in Yongcheng prefecture after a period of eleven years without a reported case [5,6]. Malaria incidence increased to 0.02 per 100,000 population in 2006, which accounted for $4.52 \%$ of the total malaria cases in China [7].

In central eastern China including Yongcheng prefecture, where malaria incidence remains seasonal and unstable, there is a need for timely confirmation of significant factors and development of factors targeting malaria interventions to curtail malaria incidence. Although studies have described malaria and its interventions in detail or factor analysis in central China $[5,6,8$ $10]$, but thus far, attempts to develop predictive models of malaria epidemics with environmental variables, which are accurate on the local scale, have not met with success.

Malaria is one of the important environmental diseases. When the environmental parameters (such as temperature, humidity) permit, Anopheles mosquitoes would transmit the pathogen, Plasmodium spp [11]. Therefore, to figure out how malaria varies due to seasonal or year-to-year changes in environmental variables is essential for the national malaria elimination plan in order for it to allow interventions to be adapted to the specific sites or times of year. Although previous studies proved that weather variables have exerted great influences on malaria incidence in different regions of the world [12-20], controversial issues still remain. First of all, some experts argued that these correlations were questionable $[20,21]$. For example, some of them claimed that rainfall had an significant effect on the incidence of malaria [22-23], whereas others did not detect a significant relationship [24,25]. Moreover, the meteorological factors that have significant statistical correlations with malaria vary greatly between geographic areas within the world, which would complicates the decision-making process in choosing weather monitoring targets [26]. In addition to those controversies, Anopheles density is considered as a proximate factor of malaria transmission while climatic indicators are distal risk factors, however, few researchers have treated mosquitoes abundance as an independent variable in statistical models to account for malaria incidence due to limited availability of entomological data [27]. Last but not the least, studies only conducted correlation analyses might have been insufficient $[24,28]$ In sum, it is necessary to perform further studies to elucidate the impacts of environmental factors, such as meteorological factors, Anopheles density on malaria incidence; this study did this based on data from Yongcheng prefecture, China as one example.

The data used in this study were collected from Yongcheng prefecture during the period between 2006 and 2010, and were analyzed using Geographic Information System (GIS) and Generalized Estimating Equations (GEE) approach. The aim was to provide not only a scientific basis for malaria monitoring and control in Yongcheng prefecture but also valuable information for malaria elimination in other areas of seasonal and unstable malaria incidence.

\section{Methods}

\section{Study area}

The site selected for the study was Yongcheng prefecture, located in eastern Henan Province (latitude $33^{\circ}$ $42^{\prime} \sim 34^{\circ} 18^{\prime} \quad \mathrm{N}$ and longitude $\left.115^{\circ} 58^{\prime} \sim 116^{\circ} 39^{\prime} \quad \mathrm{E}\right)$ (Figure 1). The prefecture has an area of $1994.49 \mathrm{~km}^{2}$ and a population of 1.46 million. Yongcheng prefecture has a temperate and monsoonal climate with four clearly distinct seasons, with monthly maximum air temperate of $27.3^{\circ} \mathrm{C}$, monthly minimum temperature of $-1.9^{\circ} \mathrm{C}$, and monthly average precipitation of $219.05 \mathrm{~mm}$. The geographic landscape and complex climate situation such as suitable temperature and humidity, abundant rainfall, and existence of water bodies, provided favourable breeding sites for Anopheles, which would contribute to making it a suitable environment for $P$. vivax malaria transmission.

\section{Data collection and management}

In China, malaria is a statutory notifiable category B infectious disease [30], reported to the National Chinese Center for Disease Control and Prevention (CDC). Monthly malaria case data from 2006 to 2010 were obtained from Yongcheng CDC. Physicians at hospitals and clinics reported suspected malaria cases to the local $\mathrm{CDC}$, and the cases were defined based on the diagnostic criteria and principles of management for malaria (GB 15989-1995) issued by Ministry of Health of the People's Republic of China. Only the cases confirmed clinically and by laboratory test, including thick and thin blood smear, were included in our study. The population 


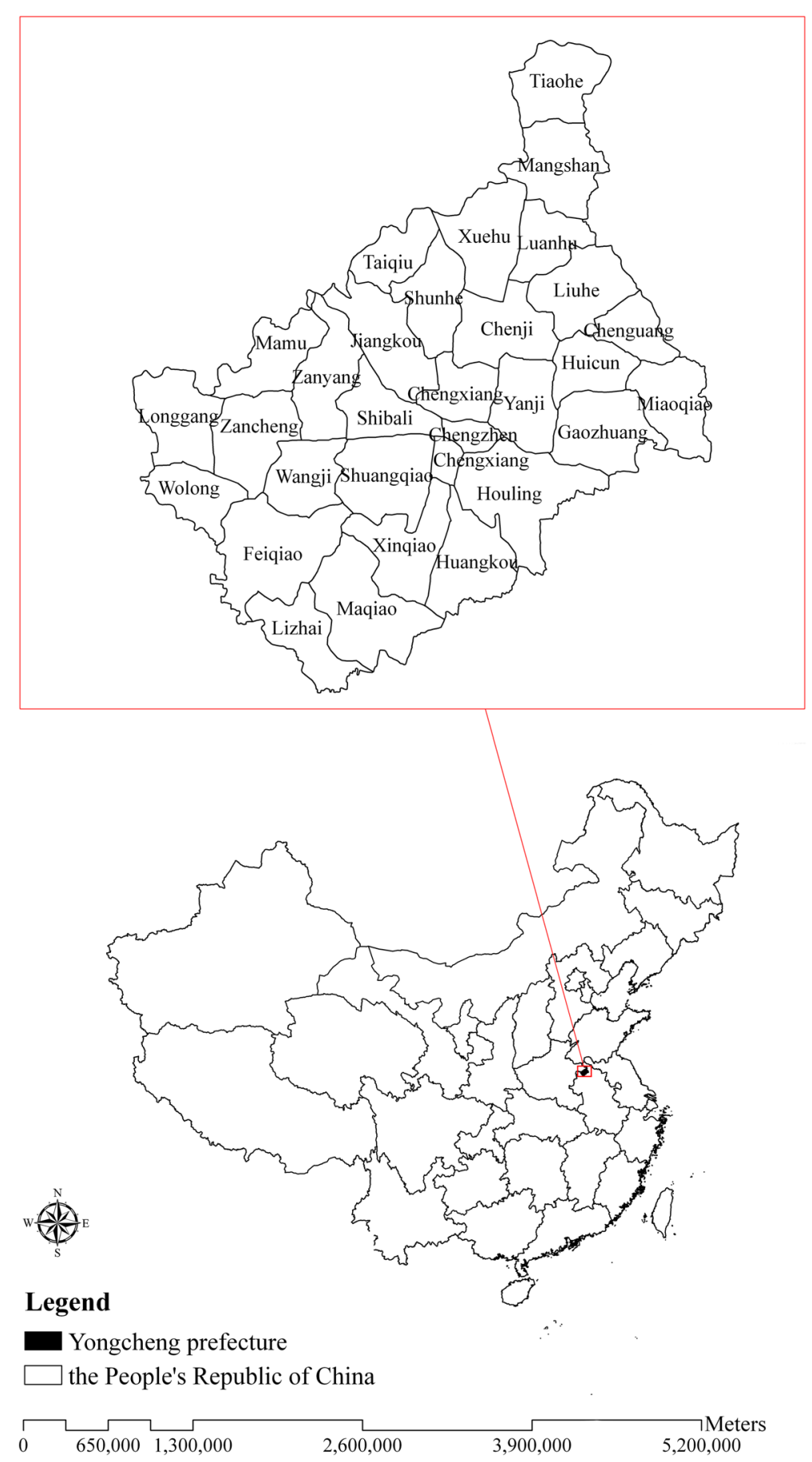

Figure 1 Location of Yongcheng prefecture in China.

data were obtained from reports produced by Yongcheng Statistical Bureau.

Monthly weather data from 2006 to 2010 were obtained from China Meteorological Data Sharing Service System (http://cdc.cma.gov.cn/), including monthly average temperature $\left(\mathrm{T}_{\mathrm{avg}}\right)$, maximum temperature $\left(\mathrm{T}_{\max }\right)$, minimum temperature $\left(\mathrm{T}_{\text {min }}\right)$, average relative humidity
$\left(\mathrm{H}_{\mathrm{avg}}\right)$, minimum humidity $\left(\mathrm{H}_{\mathrm{min}}\right)$, total 24-hour rainfall $(\mathrm{R})$, average wind velocity $\left(\mathrm{W}_{\mathrm{avg}}\right)$, maximum wind velocity $\left(\mathrm{W}_{\max }\right)$, extreme wind velocity $\left(\mathrm{W}_{\mathrm{e}}\right)$ and duration of sunshine(S). Due to the unavailability of weather data of Yongcheng prefecture, geostatistical methods of kriging was used to estimate values of meteorological parameters in Yongcheng using monthly weather 
observations measured at its eight neighbouring climatic stations, including Shangqiu, Xihua, Dangshan, Xuzhou, Haozhou, Suzhou, Fuyang, and Bengbu weather station. The distribution of those weather stations was mapped in Figure 2.

Data on Anopheles density were collected by Yongcheng CDC. Since 2005, when Maqiao village, Yongcheng prefecture was identified as a national malaria monitoring site, samples of Anopheles have been collected fortnightly from June to August using China malaria surveillance protocol [30]. Anopheles density was measured by two methods: human-bait catches and bednet trap. Human baits were positioned in outdoor areas near households and mosquito breeding sites to collect mosquitoes continuously from 7:00 PM to 10:00 PM. 50 bed-net traps were distributed to 50 households to collect indoor mosquitoes from 6:00 AM to 7:00 AM. Afterwards, the collected mosquitoes were then separated, sorted, and identified. All collected Anopheles in the study period were morphologically identified as $A n$.
Sinensis, and a sample was re-identified as An. sinensis by PCR, which is in accordance with the results of previous studies that $A n$. sinensis was the only species reported to be the vector capable of transmitting $P$. vivax in Yongcheng [5,8]. Semi-monthly An. sinensis density measured by human-bait catches $\left(D_{\text {bait }}\right)$ and bed-net trap $\left(D_{\text {net }}\right)$ was calculated as the number of mosquitoes per person"night or per 50 mosquito nets. The monthly mean value for An. sinensis density used in this study was calculated from semi-monthly data. If monthly An. sinensis density is unavailable in some month (except for June to August) of the year, we treated it as zero in the process of figure drawing and model building because of its seasonality.

\section{Ethical approval}

Ethical approval of this study was obtained from the Ethical Committee of China CDC and permission was also got from the Municipal Government, the Municipal Health Bureau and CDC in Yongcheng city.

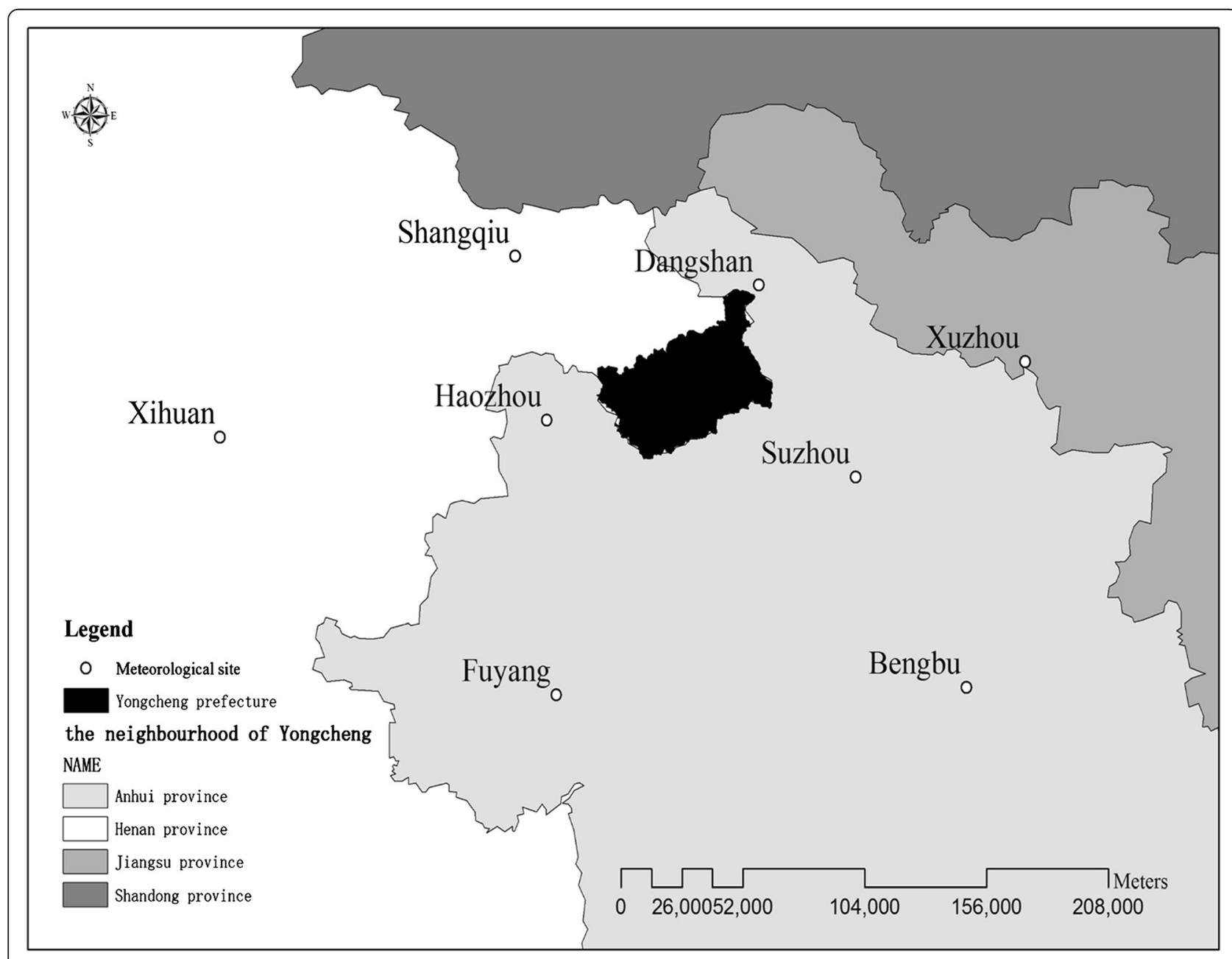

Figure 2 Positions of the eight climatic stations neighbouring Yongcheng prefecture. 


\section{Kriging analysis for spatial interpolation of meteorological factors}

Some weather values at unsampled locations were interpolated by Kriging method [31]. Monthly observed values of climatic data from eight sampled weather stations neighbouring Yongcheng prefecture was the main input variables. Kriging was performed following the procedures described by Journel et al. [32] and Burgess et al. [33], which included preliminary data analysis, structural data analysis, log kriging estimations, and image generations of spatial results. Spatially distributed values of weather factors in Yongcheng prefecture were estimated based on spherical model in this study, which was regarded as the most widely used semi-variogram model [34]. Monthly weather values at county- or prefecture-lever were calculated by using the method of Zonal statistics. The above analyses were conducted with ArcGIS 9.2 software (ESRI Inc, Redlands, California).

\section{Mapping malaria incidence and average temperature with GIS}

We conducted GIS-based analyses of the spatial distribution of yearly malaria incidence and average temperature as well as their visual correlation. The annualized average incidence of malaria per 100,000 persons and annual average temperature at each towlever over the five years (from 2006 to 2010) were calculated and mapped based a town-level polygon map of Yongcheng at 1:1000 000 scale with ArcGIS 9.2 software (ESRI Inc, Redlands, California). Regions with different intensities of malaria incidence and various values of average temperature were marked with different colours on the town-level, with higher incidence and temperature being indicated by darker colour.

\section{Temporal analysis with GEE technology}

The monthly malaria incidence, An. sinensi density, and weather variables were calculated and plotted to observe their seasonal fluctuations and correlations from 2006 to 2010.

Time varying influencing factors were treated with different time lags, from 0 - to 3 - month lags, to account for delays in their effects on malaria incidence. The lag size was determined by comparing quasi-likelihood under the independence model criterion (QIC) values in models with various lag sizes [35].

Univariate analyses were made by regressing single factors of interest against monthly malaria incidence to estimate crude associations between malaria incidences and influencing factors. Multivariable models were built to examine the effects of combinations of influencing factors on malaria incidence. Candidate factors selected for the multivariable model were determined through statistical performance of factors in the univariate analysis, and hypothesized relationships. Candidate influencing factors were inputted into the model in their presumed order of importance, and then nonstatistically significant factors were removed in their presumed inverse order of importance unless the remaining factors were deemed important for theoretical reasons at $\alpha=0.05$ level.

Two kinds of multivariable models were built up in this study using GEE approach. The difference between two models lay in whether they included malaria incidence of the previous month into the model. Model 1 was constructed to estimate the relationships between An. sinensis density, weather variables and malaria incidence. Model 2 was developed to examine the effects of An. sinensis density, weather variables and the malaria incidence of the previous month on malaria incidence.

The goodness of fit of the GEE model was measured by "marginal R-square", which was interpreted as the amount of variance in the response variables that were explained by the fitted model[36], and QIC value, which was useful in selecting an appropriate correlation structure $[8,37,38]$. The model with a lowest QIC score and a highest R-square was preferred. GEE analysis was implemented by STATA software 11.0 (Stata Corp. College Station, Texas).

\section{Results}

Spatio-temporal distribution of malaria incidence and influencing factors

From 2006 to 2010, 6,546 malaria cases were reported (7.88 cases per 100,000 population) in Yongcheng prefecture, which were all diagnosed as $P$. vivax malaria. The majority of cases were farmers (76.08\%). The annual malaria incidence at the town level showed an increasing pattern from the north to south of Yongcheng prefecture between 2006 and 2010 (Figure 3). The areas of highest malaria incidence were distributed in the southern region of Yongcheng prefecture, Maqiao village and Lizhai village, bordering northern Anhui Province. As Figure 4 showed, the yearly average temperature also rose from the north to south at the town level, with higher values distributed in the Southeast of Yongcheng prefecture.

There was a decline (15.45 cases per 100,000 population) in annual malaria incidence from 2006 to 2010, and the highest incidence occurred in 2006 with 16.72 cases per 100,000 population. Monthly P. vivax malaria incidence showed a seasonal pattern, whose peak period was from July to November, a period when nearly $86.58 \%$ of total malaria cases were reported (Figure 5). The vector specie that transmitted malaria in Yongcheng prefecture was morphologically identified as An. sinensis, with PCR results of a random sample of 30 mosquitoes confirmed as An. sinensis. An. sinensis density measured 

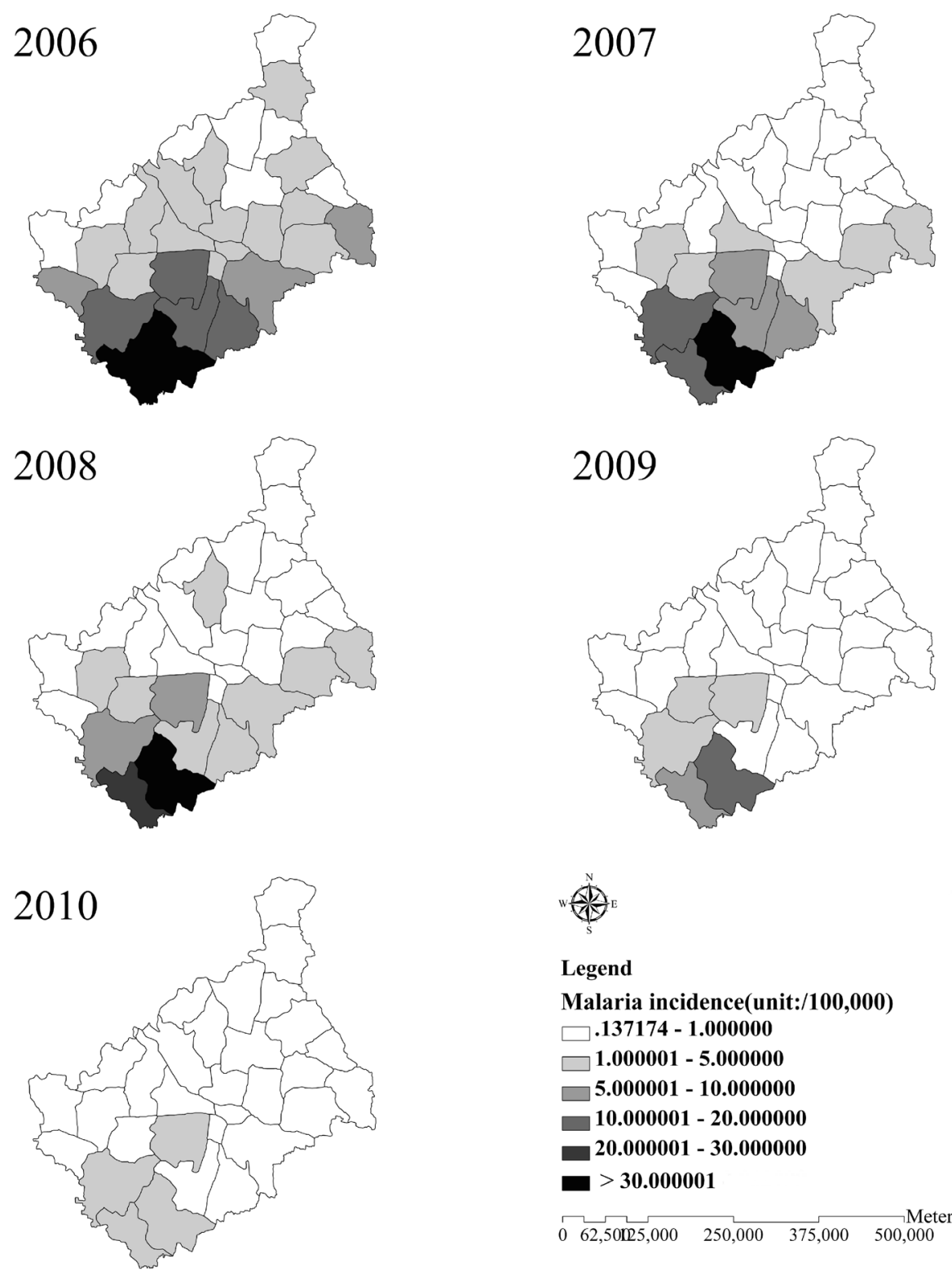

Legend

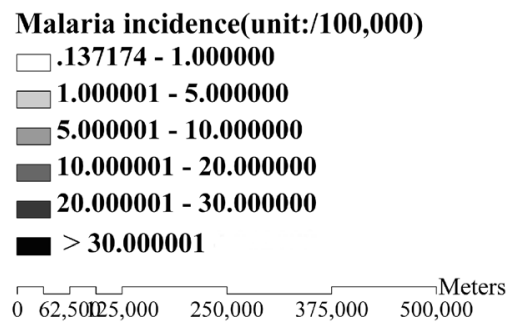

Figure 3 Annual malaria incidence at county level from 2006 to 2010 in Yongcheng prefecture, China. The county-lever areas are colour-coded according to annual malaria incidence, with higher malaria incidence distributed in the South of Yongcheng prefecture.

by different methods particularly the human-bait collections displayed a seasonal variation where the valleys and peaks closely corresponded to malaria incidence. Moreover, meteorological factors also fluctuated seasonally during the study period in addition to precipitation, showing possible relationships with malaria incidence (Figure 5).

\section{Crude correlations between malaria incidence and environmental factors}

Univariate analysis was preformed to provide a basis for selecting significant factors for malaria incidence from twelve influencing factors. Monthly An. sinensis density, temperature, humidity, and rainfall had significant positive correlations with malaria incidence with delays of zero to three months, while wind velocity showed negative correlations with delays of zero and one month. There was not a significant association observed between duration of sunshine and malaria incidence (Table 1). All these significant factors with various lag sizes were entered into the multivariable model.

\section{Model building and evaluation}

Twelve variables, such as $D_{\text {bait }}, D_{\text {net }}, T_{\text {avg }}, T_{\text {max }}, T_{\text {min }}, R$, $\mathrm{H}_{\text {avg }}, \mathrm{H}_{\min }, \mathrm{W}_{\text {avg }}, \mathrm{W}_{\max }, \mathrm{W}_{\mathrm{e}}$, and $\mathrm{S}$ were inputted into a model for variable selection. The model with $n$-binomial 

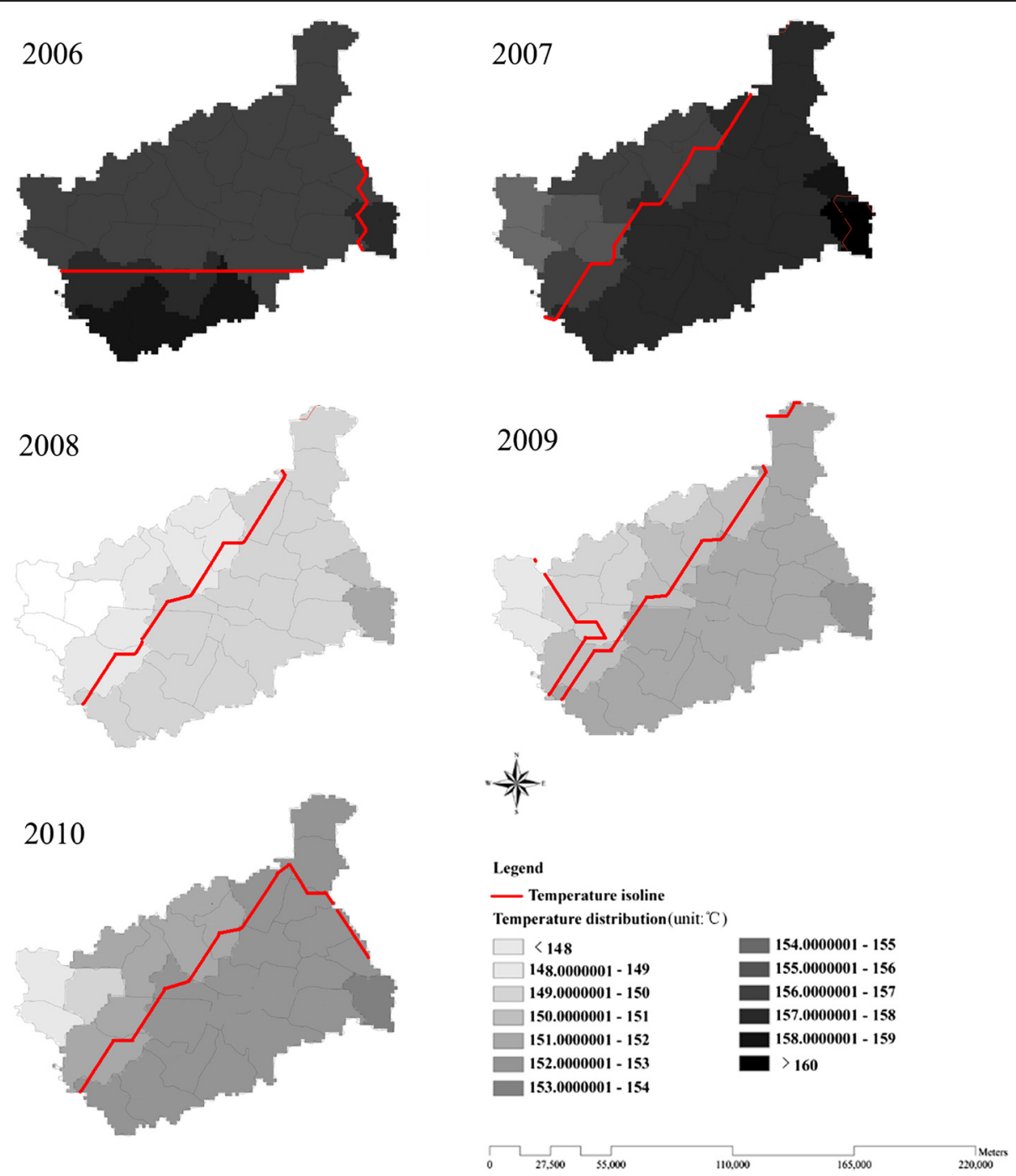

Figure 4 Yearly average temperature at county level from 2006 to 2010 in Yongcheng prefecture, China. The county-lever areas are colour-coded according to yearly average temperature, with higher temperature distributed in the Southeast of Yongcheng prefecture.

distribution (model 1) effectively explained the relationships between An. sinensis density, weather variables and malaria incidence (Table 2, model fitting QIC $=37.141$, $\left.R^{2}=0.520\right)$. Significant factors contributing to malaria incidence showed in model 1 were $D_{\text {bait }}(\beta=0.050$, $\mathrm{P}=0.033)$ at 1 -month lag, $\mathrm{T}_{\max }$ at 1 -month lag $(\beta=0.174, P<0.001)$, and $W_{e}$ at 0 -month lag $(\beta=-0.145$, $\mathrm{P}=0.009$ ) (Table 3 ). No interaction terms were significant in the multivariable model.

Meanwhile, thirteen variables, including $D_{\text {bait }}, D_{\text {net }}$, $\mathrm{T}_{\text {avg }}, \mathrm{T}_{\text {max }}, \mathrm{T}_{\text {min }}, \mathrm{H}_{\text {avg }}, \mathrm{H}_{\text {min }}, \mathrm{R}, \mathrm{W}_{\text {avg }}, \mathrm{W}_{\text {max }}, \mathrm{W}_{\mathrm{e}}, \mathrm{S}$ and the malaria incidence of the previous month $\left(\mathrm{I}_{\mathrm{p}}\right)$ were added into another model to select variables. A best-fit model (model 2) was with n-binomial distribution (Table 2, model fitting $\mathrm{QIC}=16.934, \mathrm{R}^{2}=0.818$ ), showing that $\mathrm{T}_{\max }$ at a lag of one month $(\beta=0.153, \mathrm{P}<0.001)$, $\mathrm{H}_{\text {avg }}$ at one month lag $(\beta=0.024, P=0.010)$, and $I_{p}$ $(\beta=0.049, P<0.001)$ significantly associated with malaria incidence (Table 4). However, $D_{\text {bait }}$ and $D_{\text {net }}$ failed to be included in model 2. No interaction terms were significant in the multivariable model.

Expected values fitted by model 1 or model 2 and actual observed values of malaria incidence in 2006-2010 were shown in Figures 6 and 7. Fitted values predicted by model 1 were in agreement with actual values of malaria incidence in the first three years $(F=0.645$, $\mathrm{P}=0.425$ ), but they were over-estimated to a greater extent in the last two years $(\mathrm{F}=29.052, \mathrm{P}<0.001)$. However, fitted values predicted by model 2 were in accordance with actual values of malaria incidence in the study period of 2006-2010 $(\mathrm{F}=0.032, \mathrm{P}=0.857)$.

Scatterplots of the differences between the predicted and observed malaria incidence were plotted (Figures 8 and 9), and the arithmetic mean and 95\% confidence 

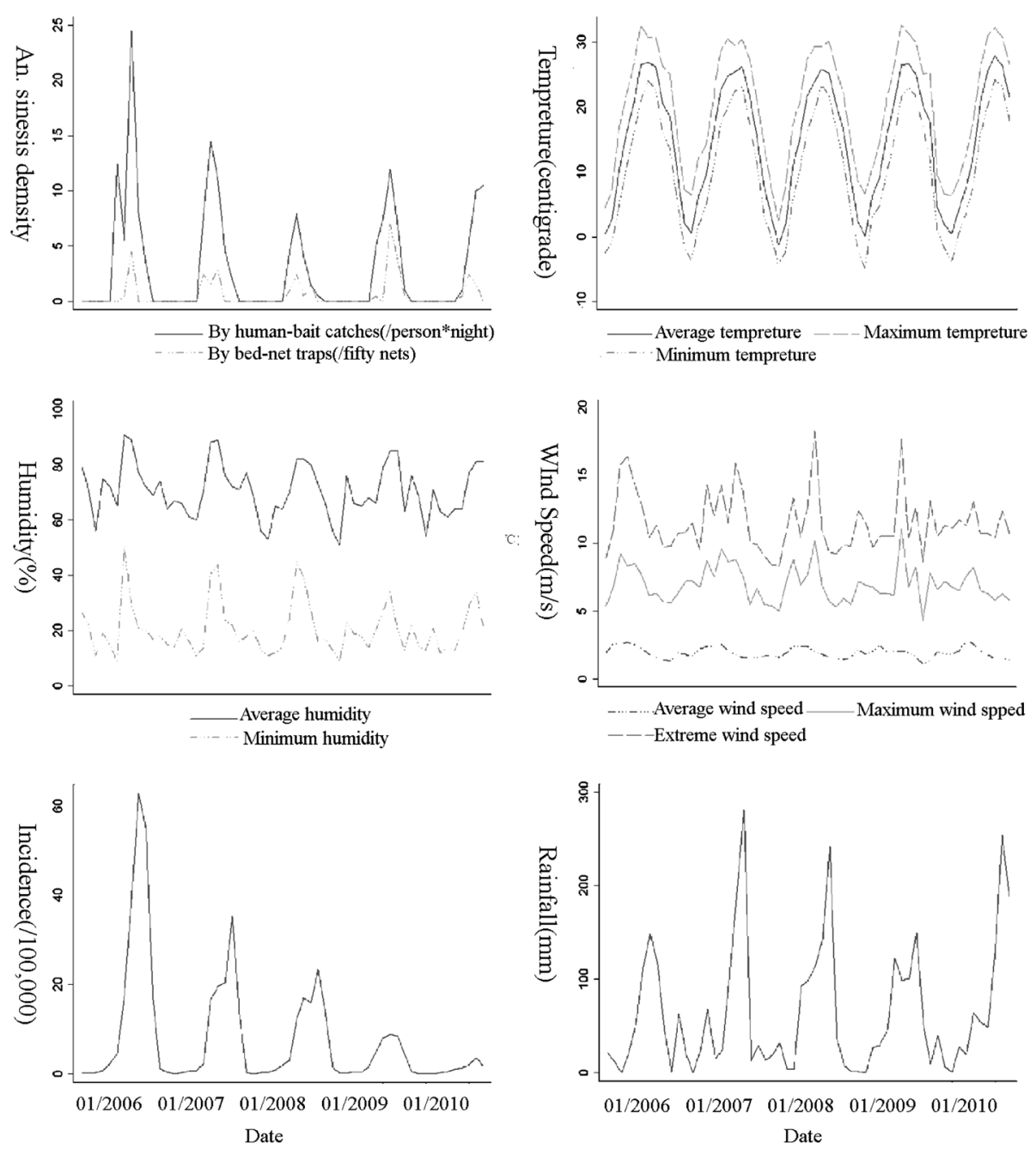

Figure 5 Time series plots of malaria incidence and influencing factors from 2006 to 2010 in Yongcheng prefecture, Henan. Influencing factors include $D_{\text {bait, }} D_{\text {net, }} T_{\text {avg, }}, T_{\max }, T_{\min }, H_{\text {avg, }}, H_{\min }, R, W_{\text {avg }}, W_{\text {max }}, W_{e}$.

interval for the differences were calculated. The average difference values between actual values and fitted values of model 1 (D-value 1 ) or model2 (D-value 2) were respectively $1.476 / 100,000$ (95\%CI: $-1.498,4.451 / 100,000)$, 0.769/100,000 (95\%CI: $-1.347,2.885 / 100,000)$. There was no significant difference between the actual values and the values predicted by model 1 or model 2 .

The best-fit model was model 2 , because it had not only a smaller D-value $(0.769 / 100,000)$, but also a smaller $\mathrm{QIC}$ value and a larger $\mathrm{R}^{2}\left(\mathrm{QIC}=16.934, \mathrm{R}^{2}=0.818\right)$, compared to model 1 ( $\mathrm{D}$-value $=1.476 / 100,000$, $\mathrm{QIC}=$ $37.141, R^{2}=0.520$ ).

\section{Discussion}

This is the first study spatially and temporally exploring the effect of weather variables and vector parameters on malaria incidence in China. The data involved in this study were relevant because all of them were obtained from national monitoring data. In this study, clear spatial heterogeneity and temporal clustering of malaria incidence could be found, with higher incidence distributed in the Southern Yongcheng spatially and in July to November temporally. The finding indicated that areas and months with higher malaria transmission risk should be focused on more public health attention and resources. Spatial heterogeneity of malaria incidence (higher incidence in the south) could be explained by the spatial variability of temperature and the distribution of malaria imported cases. First of all, Southern Yongcheng prefecture has been confronted with higher pressure from imported cases, as its was adjacent to Suixi, Guoyang, Xiao, and Huaibei county in Anhui province, which were identified as high-endemic areas of malaria (incidence $>30 / 100,000$ ) [10]. Furthermore, its spatial decreasing pattern from the north to south was somehow in accordance of temperature change, particularly 
Table 1 Coefficients of univariate analysis among environmental factors on $\mathrm{P}$. vivax malaria incidence

\begin{tabular}{|c|c|c|c|c|c|c|c|c|c|c|c|c|c|}
\hline $\begin{array}{l}\text { Lag-time } \\
\text { (months) }\end{array}$ & $\begin{array}{l}\text { Distribution } \\
\text { type }\end{array}$ & $D_{\text {bait }}$ & $D_{\text {net }}$ & $\mathrm{T}_{\text {avg }}$ & $T_{\max }$ & $T_{\min }$ & $\mathrm{H}_{\mathrm{avg}}$ & $\mathrm{H}_{\min }$ & $\mathbf{R}$ & $W_{\text {avg }}$ & $\mathrm{W}_{\max }$ & $w_{e}$ & $S$ \\
\hline \multirow[t]{2}{*}{0} & Poisson & $0.091^{* * *}$ & 0.164 & $0.074^{* * *}$ & $0.078^{* * *}$ & $0.073^{* * *}$ & $0.073^{* * * *}$ & $0.044^{* * *}$ & 0.002 & $-2.782^{* * *}$ & $-0.453^{* *}$ & -0.201 & -0.005 \\
\hline & n-binomial & $0.144^{* * *}$ & 0.186 & $0.122^{* * *}$ & $0.113^{* * *}$ & $0.110^{* * *}$ & $0.107^{* *}$ & $0.068^{* * *}$ & 0.002 & $-3.180^{* * *}$ & $-0.391^{*}$ & $-0.167^{* *}$ & -0.010 \\
\hline \multirow[t]{2}{*}{1} & Poisson & $0.120^{* * *}$ & $0.250^{*}$ & $0.143^{* * *}$ & $0.148^{* * *}$ & $0.137^{* * *}$ & $0.097^{* * *}$ & $0.059^{* * *}$ & $0.006^{* *}$ & -1.782 & -0.185 & -0.040 & -0.003 \\
\hline & n-binomial & $0.222^{* * *}$ & $0.370^{* *}$ & $0.192^{* * *}$ & $0.199 * * *$ & $0.188^{* * *}$ & $0.110^{* * *}$ & $0.073^{* * *}$ & $0.008^{* *}$ & $-2.073^{* * *}$ & -0.150 & -0.035 & -0.00 \\
\hline \multirow[t]{2}{*}{2} & Poisson & $0.116^{* * *}$ & $0.248^{*}$ & $0.184^{* * *}$ & $0.188^{* * *}$ & $0.167^{* * *}$ & $0.086^{* * *}$ & $0.064^{* * *}$ & $0.009^{* * *}$ & -0.320 & 0.121 & 0.109 & 0.003 \\
\hline & n-binomial & $0.173^{* * *}$ & $0.398^{* * *}$ & $0.181^{* * *}$ & $0.185^{* * *}$ & $0.175^{* * *}$ & $0.067^{* *}$ & 0.049 & $0.015^{* * *}$ & -0.404 & 0.126 & 0.128 & 0.002 \\
\hline \multirow[t]{2}{*}{3} & Poisson & $0.081^{* *}$ & 0.079 & $0.124^{* * *}$ & $0.130^{* * *}$ & $0.111^{* * *}$ & 0.039 & 0.039 & $0.007^{* * *}$ & $0.950^{*}$ & $0.469^{* * *}$ & $0.199^{* * *}$ & 0.009 \\
\hline & n-binomial & $0.105^{* *}$ & 0.130 & $0.128^{* * *}$ & $0.130^{* * *}$ & $0.124^{* * *}$ & 0.032 & 0.023 & $0.012^{* * *}$ & $1.392^{* * *}$ & $0.324^{* *}$ & $0.277^{* * *}$ & 0.00 \\
\hline
\end{tabular}

("*": The regression coefficient is significant at level of $a=0.05$, "***": The regression coefficient is significant at level of $a=0.01$, "***": The regression coefficient is significant at level of $a=0.001$ ).

in 2006. Only temperature was selected to assess the influencing factor associated with spatial heterogeneity owing to the founding from previous studies that temperature might be a major determinant of malaria incidence in China [26]. Therefore, public resource should be allocated proportionally in different areas based on the risk predicted by imported possibility and average temperature, and close collaboration should be established between Henan and Anhui to effectively control and prevent malaria together. From the perspective of time, annual malaria incidence in Yongcheng prefecture showed an obvious decrease from 2006 to 2010. However, prevention and control of malaria should not be taken slightly, because Yongcheng prefecture is still at risk for malaria due to the existing of An. sinensis, suitable weather condition for the growing of An. sinensis and Plasmodium, and the risk has been possibly varying with weather changes and population movement $[39,40]$. Determining the principal influencing factors of malaria incidence would be beneficial for malaria risk assessment and thus providing a basis for the policy making for malaria control technologies.

Malaria is a vector-borne infectious disease, and as such, is sensitive to environmental change [40-42]. Anopheles density, as a proximate environmental factor of malaria transmission, play an important role in estimating and predicting malaria risk [27]. Climatic variables have also been established as important environmental drivers of malaria transmission [43], because of their impacts on the growth and reproduction rates of mosquitoes, the temporal activity pattern of the population as well as the life cycle of Plasmodium [44-47].

Table 2 Comparison of GEE models

\begin{tabular}{llllll}
\hline & \multicolumn{3}{c}{ Model 1 } & & \multicolumn{2}{c}{ Model 2 } \\
\cline { 2 - 3 } & QIC & QIC_u & & QIC & QIC_u \\
\hline Poisson Distribution & 131.941 & 119.726 & & 351.132 & 319.353 \\
n-binomial Distribution & 16.934 & 23.029 & & 37.141 & 41.236 \\
\hline
\end{tabular}

The best-fit model (model 2) derived from the study was reliable and had a good fit and predictive validity $\left(\mathrm{QIC}=16.934, \quad \mathrm{P}<0.001, \quad \mathrm{R}^{2}=0.818\right)$, which provided insights into the most important drivers of $P$. vivax malaria, including maximum temperature, average humidity and incidence of previous month that influenced seasonal fluctuation of $P$. vivax malaria incidence. The result that temperature rise would contribute to malaria transmission was in agreement with some researchers [46-52], although there were still some other researchers who argued that this relationship was not significant $[19,53]$, or that it was uncertain [54]. It has been demonstrated that temperature increase would improve the survival chances of Anopheles and thus contribute to the malaria transmission $[55,56]$ Moreover, relative humidity exerted an influence on the survival of mosquito eggs and adults and the moderate increase in malaria risk associated with average humidity observed in this study was consistent with previous findings [57]. Conversely, some literature found a correlation between rainfall and malaria [49], while other studies found no correlation [51]. In this study, rainfall failed to enter the best-fit model as a predictor of malaria epidemics. The phenomenon could be explained by the complex nonlinear association between rainfall and malaria incidence. Rainfall is beneficial to the growth and reproduction of

Table 3 Poisson and $\mathbf{n}$-binomial regression of malaria incidence on influencing factors (the result of Model 1)

\begin{tabular}{|c|c|c|c|c|c|c|c|}
\hline \multirow{2}{*}{$\begin{array}{l}\text { Lag-time } \\
\text { (months) }\end{array}$} & \multirow{2}{*}{ Variables } & \multicolumn{3}{|c|}{ Poisson Distribution } & \multicolumn{3}{|c|}{ n-binomial Distribution } \\
\hline & & $\beta$ & SE & $P$ & $\beta$ & SE & $P$ \\
\hline 1 & $D_{\text {bait }}$ & 0.038 & 0.017 & 0.025 & 0.05 & 0.023 & 0.033 \\
\hline 1 & $T_{\max }$ & 0.096 & 0.017 & $<0.001$ & 0.174 & 0.015 & $<0.001$ \\
\hline 1 & $\mathrm{H}_{\mathrm{avg}}$ & 0.044 & 0.011 & $<0.001$ & & & \\
\hline \multirow[t]{2}{*}{0} & $W_{e}$ & & & & -0.145 & 0.055 & 0.009 \\
\hline & constant & -3.811 & 0.958 & $<0.001$ & -0.975 & 0.726 & 0.179 \\
\hline
\end{tabular}

(Coef: regression coefficient, SE: estimated regression coefficient standard error). 
Table 4 Poisson and $\mathbf{n}$-binomial regression of malaria incidence on influencing factors (the result of Model 2)

\begin{tabular}{|c|c|c|c|c|c|c|c|}
\hline \multirow{2}{*}{$\begin{array}{l}\text { Lag-time } \\
\text { (months) }\end{array}$} & \multirow{2}{*}{ Variables } & \multicolumn{3}{|c|}{ Poisson Distribution } & \multicolumn{3}{|c|}{ n-binomial Distribution } \\
\hline & & $\beta$ & SE & $P$ & $\beta$ & SE & $P$ \\
\hline 1 & $T_{\max }$ & 0.137 & 0.019 & $<0.001$ & 0.153 & 0.012 & $<0.001$ \\
\hline 1 & $\mathrm{H}_{\mathrm{avg}}$ & & & & 0.024 & 0.009 & 0.01 \\
\hline 2 & $\mathrm{R}$ & 0.005 & 0.001 & $<0.001$ & & & \\
\hline \multirow[t]{2}{*}{0} & $I_{p}$ & 0.039 & 0.003 & $<0.001$ & 0.049 & 0.006 & $<0.001$ \\
\hline & constant & -2.51 & 0.488 & $<0.001$ & -4.449 & 0.589 & $<0.001$ \\
\hline
\end{tabular}

(Coef: regression coefficient, SE: estimated regression coefficient standard error).

mosquito if it is moderate, because it often leads to puddles and increased local humidity; however, excessive rain can also wash away eggs and completely destroy breeding sites [58]. This result indicated that it was not necessary to consider rainfall as a predictor in Yongcheng, which made malaria surveillance simpler in this area.

In addition to factors mentioned above, although $A n$. sinensis densities failed to enter into best-fit model (model 2), $\mathrm{D}_{\text {bait }}$ was included in model 1. The reasons why $D_{\text {bait }}$ rather than $D_{\text {net }}$ were included in model 1 probably lay in two aspects. Firstly, An. sinensis is slightly exophagic (biting outdoors) [59], and thus $\mathrm{D}_{\text {bait }}$ is more representative of malaria transmission in Yongcheng. Secondly, most of the malaria cases in Yongcheng were farmers, and they would like to sleep and work outdoors in the summer without effective protections, thus having more opportunities to be infected $[5,9]$.
Therefore, $D_{\text {bait }}$ would contribute more to the prediction of malaria incidence. Moreover, comparing the time series plots of $D_{\text {bait }}$ (Figure 5), expected values predicted by model 1 (Figure 6) and actual values (Figure 6), we found out expected values were over-estimated in 2009 and 2010 when actual malaria incidence was relatively low, which mainly due to the existence of predictor $D_{\text {bait }}$ reasoned by their similar rising tendency. It could be concluded that An. sinensis density probably had its shortcomings as a routine monitoring and predicting index. An. sinensis density may be a better predictor of malaria incidence when transmission is relatively high, as many of the female mosquitoes may have been infected by Plasmodium, and increase in An. sinensis density would lead to a direct rise in malaria incidence. However, when the incidence is at a low level, most of the female mosquitoes are possibly free of Plasmodium. In this circumstance, when a healthy human being is bitten by a female An. sinensis the probability of infection by Plasmodium is low. Therefore, we concluded that rise in An. sinensis density would possibly contribute little to the increase of malaria incidence in low transmission areas, which agreed with the result of another study that malaria transmission potential would be very low in spite of a high human biting rate in unstable malaria areas [60]. Therefore, control target should vary with the severity of malaria epidemics. When malaria incidence is high, public resource allocation should be focused on mosquito control and elimination; however, when malaria incidence is low, the key control point should lie in controlling sources of infections. Furthermore, it is

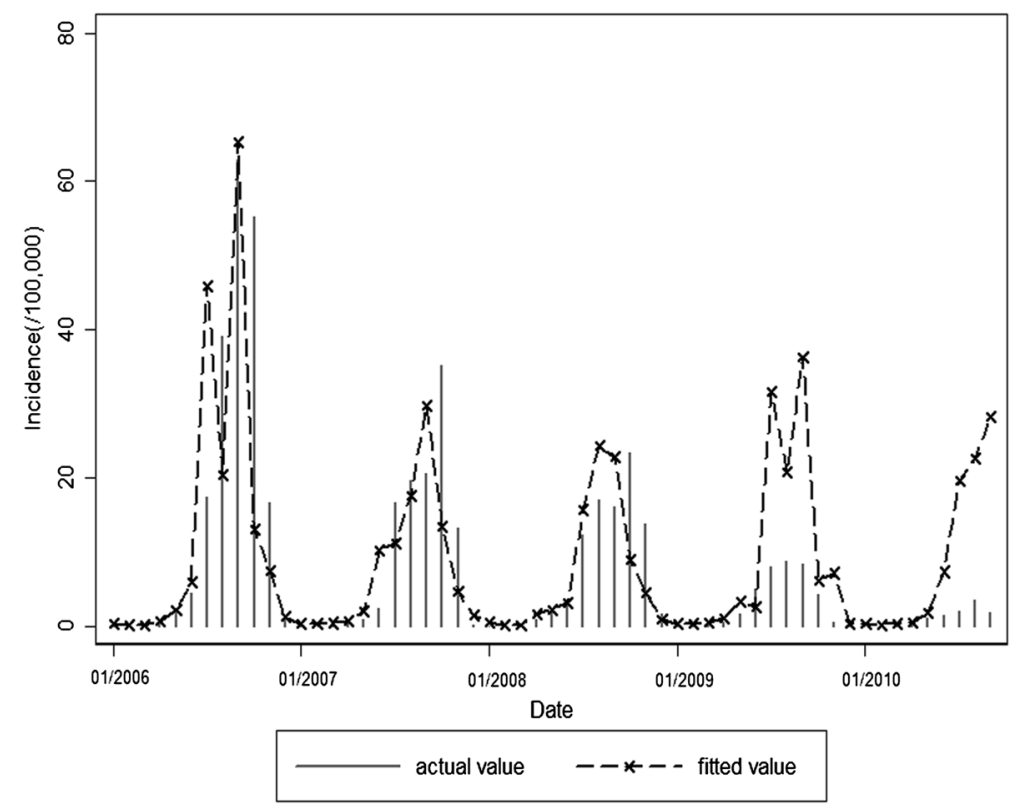

Figure 6 Expected values of model 1 and observations for malaria incidence in the study period of 2006-2010. 


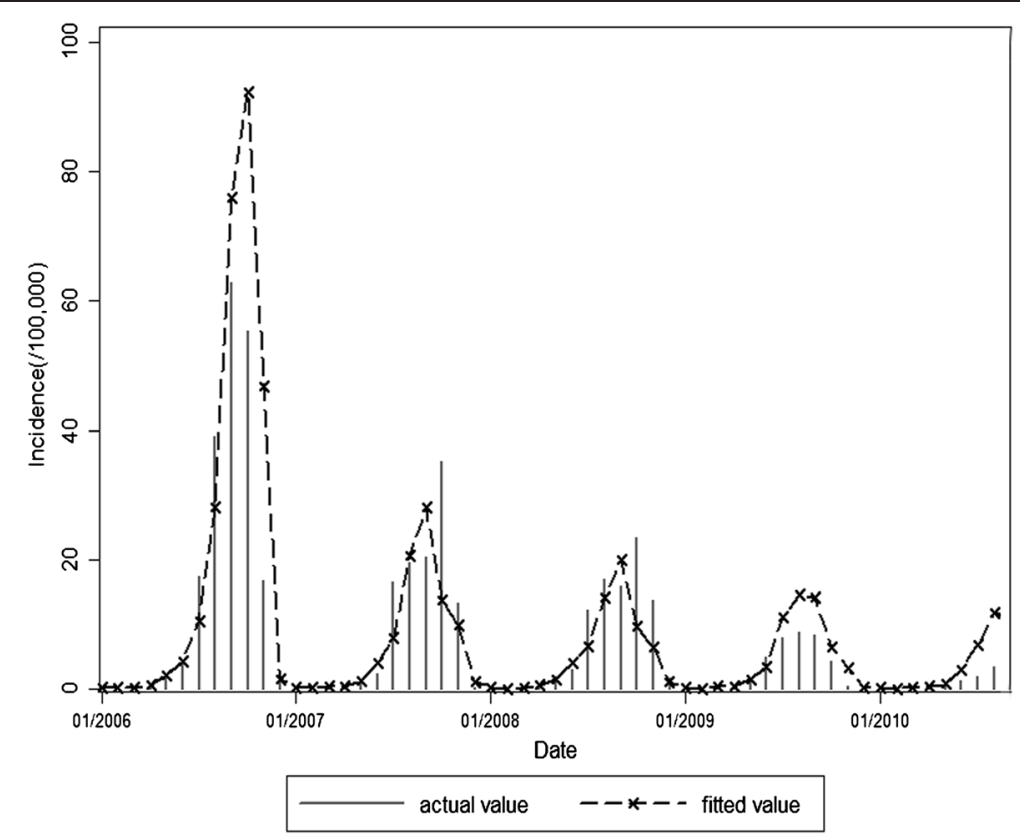

Figure 7 Expected values of model 2 and observations for malaria incidence in the study period of 2006-2010.

necessary to find a substitute, such as entomological infection rate (EIR), which can overcome the weakness of An. sinensis density as an indicator for malaria surveillance and prediction, although it can be used as a good index for predicting malaria potential risk as a previous study showed [40].

As far as the lag effect was concerned, this study found significant one month lag effects of entomological and meteorological variables on malaria incidence, and this finding was supported by several earlier studies $[17,19,35,49]$. This phenomenon could be explained by

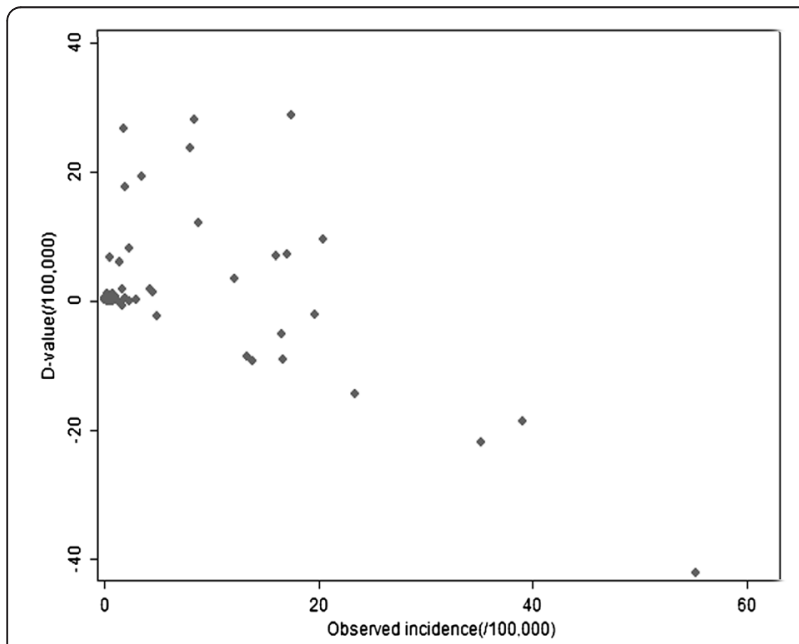

Figure 8 Scatterplot of difference between expected values predicted by model 1 and observed values of malaria incidence in the study period of $2006-2010$. the approximately one month duration of malaria infection cycle. The time incorporates several processes above. An adult mosquito first bites an infected human, and then the parasite develops in the adult mosquito (Extrinsic Incubation Period). Ten days later when the $P$. vivax sporozoites move the salivary glands, the mosquito transmits malaria to a human when it takes another blood meal. Once the person is infected, time to development of malaria symptoms and infectivity (Intrinsic Incubation Period) takes about another 1-2 weeks $[40,61]$. Knowing the approximate lag size of effects on

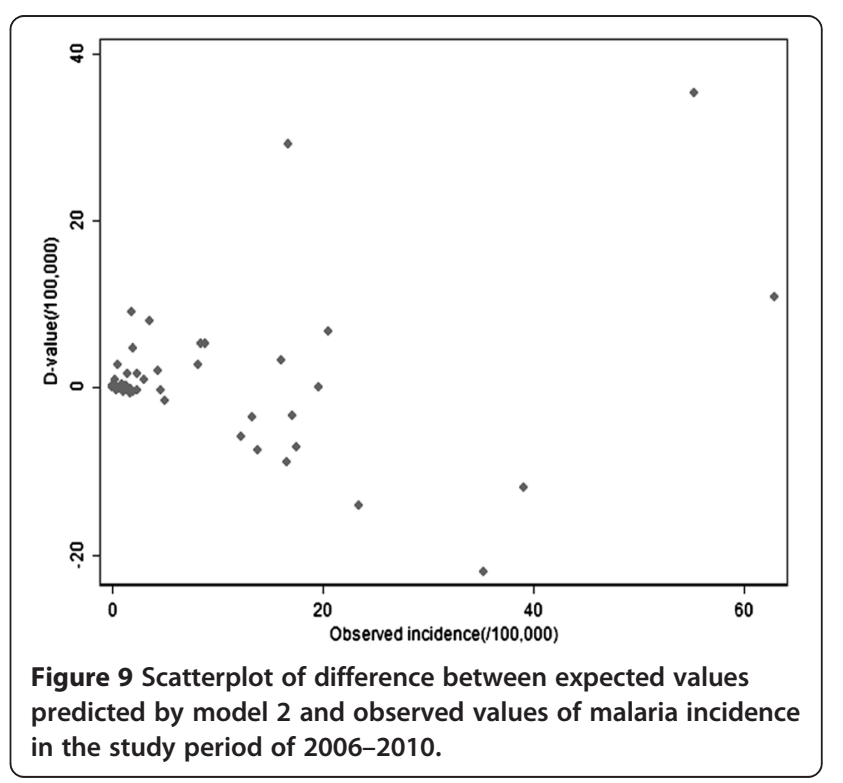


malaria incidence would benefit us to get prepared for quick and effective response on malaria epidemic easily at least one month in advance.

The transmission of malaria is complicated, and we still need further research to figure it out. For example, the temporal variation in malaria incidence could be also partially explained by continuous and effective control efforts Chinese local and national health agencies made, such as treatment in the rest period of malaria(conducted from 2004) [62] and comprehensive vector control action characterized by biological larviciding and residual spray(conducted from 2007) [8]. Human interventions are failed to be inputted into the predictive model in this study because it is difficult to measured and quantify.

\section{Conclusions}

Areas and months with higher malaria transmission risk should be focused on more public health attention and resources. The model developed in this study successfully predicted the expected incidences of malaria based on historical malaria epidemics and a combination of weather factors at one month lag, which would simplify malaria surveillance by targeting control of malaria more effectively. Furthermore, we concluded that (1)more effective indicators, such as weather variables and Plasmodium infection rate of mosquitoes should be considered to further optimize current malaria monitoring and control methods; (2)and malaria control targets should vary with intensity of malaria incidence, with more public resource allocated to control the source of infections instead of large scale An. sinensis control when malaria incidence was at a low level, which would benefit for optimizing the malaria surveillance project in China, and also could be used for the malaria monitoring and earlywarning in some other countries with unstable or low malaria transmission.

\section{Competing interest}

The authors declare that no competing interests exist.

\section{Authors' contributions}

YZ conceived this study and was involved in manuscript drafting. QYL participated in the design of the study, and helped in drafting the manuscript. RSL was involved in the study design and manuscript modification. XBL, GCZ, JYJ, HSL, and ZFL took part in the data collection and entry. All authors have read and approved the submitted version of the manuscript.

\section{Acknowledgements}

We would thank the following people: Weidong Gu, Hualiang Lin, Matthew Baylis, Jingyi Duan, and Lindsay Morton for their great contributions in compiling the historical data; the anonymous reviews for their valuable comments and suggestions. This study was supported by the National Basic Research Program of China (973 Program) (Grant No.2012CB955500, 2012CB955504) and the National Science Foundation of China (NSFC) grant (30972563).

\section{Author details}

'State Key Laboratory for Infectious Diseases Prevention and Control, National Institute for Communicable Disease Control and Prevention, Chinese Center for Disease Control and Prevention, Beijing, People's Republic of China. ${ }^{2}$ Fengtai Center for Disease Control and Prevention, Beijing, People's Republic of China. ${ }^{3}$ China CDC Key Laboratory of Surveillance and Early-Warning on Infectious Disease, Beijing, People's Republic of China. ${ }^{4}$ Shandong University Climate Change and Health Center, Jinan, People's Republic of China. ${ }^{5}$ Department of Epidemiology, West China School of Public Health, Sichuan University, Chengdu, People's Republic of China.

${ }^{6}$ Yongcheng Center for Disease Control and Prevention, Yongcheng prefecture, People's Republic of China.

Received: 13 December 2011 Accepted: 30 June 2012

Published: 23 July 2012

\section{References}

1. World Malaria Report. Geneva: World Health Organization; 2010. http:// whalibdoc.who.int/publications/2010/9789241564106_eng.pdf.

2. Krishnadas N: Millennium development goals. Global \& Indigenous Health 2009, 1:36-38.

3. The 2010-2020 plan of national malaria elimination in China. http://www. moh.gov.cn/publicfiles/business/htmlfiles/mohbgt/s10788/201005/47529. $\mathrm{htm}$.

4. Global Malaria Action Pan. http://www.rollbackmalaria.org/gmap/2-1.html.

5. Zhang HW, Su YP, Zhou GC, Liu Y, Cui J, Wang ZQ: Remerging malaria in Yongcheng city of Henan province [in China]. Chin J Vector Bio \& Control 2007, 18(1):42-44.

6. Zhou GC, Zhang HW, Su YP, Zhou SS, Huang F: Epidemiologic Analysis of Malaria Outbreak in Yongcheng prefecture County of Henan Province in 2006 [in China]. Chin J Parasitol Parasit Dis 2008, 8(4):381-383.

7. Zhou SS, Wang Y, Tang LH: Malaria Situation in the People's Republic of in 2006[in China]. Chin J Parasitol Parasit Dis 2007, 25(6):439-441.

8. Zhou GC, Huang F, Zhou SS, Zhang HW, Su YP: Malaria situation and evaluation on different malaria prevention and control measures in Yongcheng city [in China]. Chin J of Parasit Bio 2009, 4(6):449-452.

9. Sheng HF, Zhao ZH, Guo Q, Dai J: Analysis on the socio-behavioral factors related to re-emergence of malaria in Anhui province [in China]. Disease Surveillance 2010, 25(3):216-219.

10. Zhang WY, Wang $L P$, Fang $L Q, M a ~ J Q$, Xu YF, Jiang JF, Hui FM, Wang JJ, Song $L$, Yang $H$, et al: Spatial analysis of malaria in Anhui province, China. Malar J 2008, 7:206-216.

11. Thomson MC, Conor SJ, Alessandro UD, Rowlingson B, Diggle P, Cresswell $M$, Greenwood B: Predicting malaria infection in Gambian children from satellite data and bed net use surveys: the importance of spatial correlation in the interpretation of results. AmJTrop Med Hyg 1999, 61(1):2-8,

12. Pascual M, Cazelles B, Bouma MJ, Chaves LF, Koelle K: Shifting patterns: malaria dynamics and rainfall variability in an African highland. Proc Bio Sci 2008, 275(1631):123-132.

13. Alsop Z: Malaria returns to Kenya's highlands as temperatures rise. Lancet 2007, 370:925-926.

14. Olson SH, Gangnon R, Elguero E, Durieux L, Guegan JF, Foley JA, Patz JA: Links between climate, malaria, and wetlands in the Amazon Basin. Emerg Infect Dis 2010, 15:659-662.

15. Clements AC, Barnett AG, Cheng ZW, Snow RW, Zhou GC: Space-time variation of malaria incidence in Yunnan province, China. Malar J 2010, 8:180.

16. Dantur Juri MJ, Zaidenberg M, Claps GL, Santana M, Almiron WR: Malaria transmission in two localities in north-western Argentina. Malar J 2010, 8:18.

17. Pemola Devi N, Jauhari RK: Climatic variables and malaria incidence in Dehradun, Uttaranchal, India. J Vect Borne Dis 2006, 43:21-28.

18. Zacarias $\mathrm{OP}$, Andersson M: Mapping malaria incidence distribution that accounts for environmental factors in Maputo Province - Mozambique, Zacarias and Andersson. Malar J 2010, 9:79-89.

19. Gomez-Elipe A, Otero A, Herp MV, Aguirre-Jaime A: Forecasting malaria incidence based on monthly case reports and environmental factors in Karuzi, Burundi, 1997-2003. Malar J 2007, 6:129-139. 
20. Hay SI, Shanks GD, Stern DI, Snow RW, Randolph SE, Rogers DJ: Climate variability and malaria epidemics in the highlands of East Africa. Trends Parasitol 2005, 21(2):52-53.

21. Hay SI, Cox J, Rogers DJ, Randolph SE, Stern DI, Shanks GD, Myers MF, Snow RW: Climate change and the resurgence of malaria in the East African highlands. Nature 2002, 415(21):905-909.

22. Zhou G, Minakawa N, Githeko A, Yan G: Association between climate variability and malaria epidemics in the East African highlands. Proc Natl Acad Sci USA 2004, 101(8):2375-2380.

23. Kuhn K, Campbell-Lendrum D, Armstrong B, Davies C: Malaria in Britain: past, present, and future. Proc Natl Acad Sci USA 2003, 100(17):9997-10001.

24. van der Hoek W, Konradsen F, Perera D, Amerasinghe P, Amerasinghe F: Correlation between rainfall and malaria in the dry zone of Sri Lanka. Ann Trop Med Parasitol 1997, 91(8):945-949.

25. Singh N, Sharma V: Patterns of rainfall and malaria in Madhya Pradesh, central India. Ann Trop Med Parasitol 2002, 96(4):349-359.

26. Xiao D, Long Y, Wang SQ, Fang LQ, Xu DZ, Wang GZ, Li L, Cao WC, Yan YP: Spatiotemporal distribution of malaria and the association between its epidemic and climate factors in Hainan, China. Malar J 2010, 9:185-196.

27. Trape JF, Evelyne LZ, Legros F, Ndiaye G, Bouganali H, Druilhe P, Salem G: Vector Density Gradients and the Epidemiology of Urban Malaria in Dakar, Senegal. AmJTrop Med Hyg 1992, 47:181-189.

28. Bouma M, Poveda G, Rojas W, Chavasse D, Quinones M, Cox J, Patz J: Predicting high-risk years for malaria in Colombia using parameters of El Nino Southern Oscillation. Trop Med Int Health 1997, 2(12):1122-1127.

29. NPCSC (The Standing Committee of the National People's Congress): Law of the people's Republic of China on Prevention and Treatment of Infectious Diseases. http://www.moh.gov.cn/publicfiles/business/htmlfiles/mohjbyfkzj/ s3576/200804/29124.htm

30. National Malaria Surveillance Project (for Trial Implementation). http://www. moh.gov.cn/publicfiles/business/htmlfiles/mohbgt/pw10506/200804/27350. htm.

31. Holdaway MR: Spatial modeling and interpolation of monthly temperature using kriging. Clim Res 1996, 6:215-225.

32. Hudnall WH, Sigua GC, Travis RE: The physical, chemical, and mineralogical properties of Louisiana coastal marshes. In report of projects for 1989. Baton Rouge, LA: Dept Agronomy, LSU; 1989.

33. Burgess TM, Webster R: Optimal interpolation and isarithmic mapping of soil properties-I the semi-variogram and punctual kriging. J Soin Sci 1980, 31:315-331.

34. Goovaerts P: Geostatistical approaches for incorporating elevation into the spatial interpolation of rainfall. J Hydrol 2000, 228:113-129.

35. Ballinger GA: Using generalized estimating equations for longitudinal data analysis. Organ Res Methods 2004, 7(2):127-150.

36. Ziegler A: Generalized Estimating Equations. New York: Springer; 2011

37. Hwang $\mathrm{H}$, Takane $\mathrm{Y}$ : Estimation of growth curve models with structured error covariance by generalized estimating equations. Behaviormetrika 2005, 32(2):155-163.

38. Cui J, Qian G: Selection of working correlation structure and best model in GEE analyses of longitudinal data. Communications in Statisticssimulation and Computation 2007, 36(5):987-996.

39. Li ZH: Analysis of main factors associated with the prevalence of malaria in Yunnan Province at present. China Trop Med 2006, 6:1383-1384.

40. Martens P: Health and Climate Change: modelling the impacts of global warming and ozone depletion. London: Earth Scan Publications Ltd; 1998.

41. $\mathrm{Li} \mathrm{ZH}$ : Analysis of main factors associated with the prevalence of malaria in Yunnan Province at present [in China]. Chin Trop Med 2006, 6(8):1383-1384.

42. Martens P, Kovats RS, Nijhof S, Vries P, Livermore MTJ, Bradley DJ, Cox J, McMichael AJ: Climate change and future populations at risk of malaria. Global Environ Change 1999, 9(suppl 1):89-107.

43. Yé $Y$, Valérie R L, Séraphin S, Sauerborn R: Effect of meteorological factors on clinical malaria risk among children: an assessment using villagebased meteorological stations and community-based parasitological survey. BMC Publ Health 2007, 7:101-111.

44. Craig MH, Kleinschmidt I, Nawn JB, Sueur DL, Sharp BL: Exploring 30 years of malaria case data in Kwazulu-Natal, South Africa: Part I. The impact of climatic factors. Trop Med Int Health 2004, 9(12):1247-1257.

45. Koenraadt CJM, Githeko AK, Takken W: The effects of rainfall and evapotranspiration on the temporal dynamics of Anopheles gambiae s.s. and Anopheles arabiensis in a Kenyan village. Acta Trop 2004, 90(2):141-153.

46. Depinay JMO, Mbogo CM, Killeen G, Knols B, Beier J, Carlson J, Dushoff J, Billingsley $P$, Mwambi $H$, Githure J, et al: A simulation model of African Anopheles ecology and population dynamics for the analysis of malaria transmission. Malar J 2004, 3:29-50.

47. Hosen MB, Morse AP: A weather-driven model of malaria transmission. Malar J 2004, 3:32

48. Kristan M, Abeku TA, Beard J, Okia M, Rapuoda B, Sang J, Cox J: Variations in entomological indices in relation to weather patterns and malaria incidence in East African highlands: implications for epidemic prevention and control. Malar J 2008, 7(1):231-242.

49. Zhang Y, Bi P, Hiller JE: Meteorological variables and malaria in a Chinese temperate city: A twenty-year time-series data analysis. Environ Int 2010, 36(5):439-445

50. Bi P, Tong S, Donald K, Parton KA, Ni J: Climatic variables and transmission of malaria: a 12-year data analysis in Shuchen County, China. Public Health Rep 2003, 118(1):65-71.

51. Wang L, Fang L, Xu X, Wang J, Ma J, Cao W, Jin S: Study on the determinants regarding malaria epidemics in Anhui province during 2004-2006 [in China]. Chin J Epidemiol 2009, 30(1):38-41.

52. Wen L, Shi R, Fang L, Xu D, Li C, Wang Y, Yuan Z, Zhang H: Spatial epidemiological study on malaria epidemics in Hainan province [in China]. Chin J Epidemiol 2008, 29(6):581-585.

53. Tian L, Bi Y, Ho SC, Liu W, Liang S, Goggins WB, Chan EY, Zhou S, Sung JJ: One-year delayed effect of fog on malaria transmission: a time-series analysis in the rain forest area of Mengla County, south-west China. Malar J 2008, 7:110.

54. Tanser FC, Sharp B, Sueur Dl: Potential effect of climate change on malaria transmission in Africa. Lancet 2003, 362(9398):1792-1798.

55. Loevinsohn ME: Climatic change and malaria: exploring the risks. Med War 1995, 11:202-213.

56. Tong SL, Ying LV: Global Climate Change and Epidemic Disease [in China]. J Dis Cont 2000, 4(1):17-19.

57. Hui FM, Xu B, Chen ZW, Cheng X, Lu L, Huang HB, Fang LQ, Yang H, Zhou $\mathrm{HN}$, Yang HL, et al: Spatio-Temporal Distribution of Malaria in Yunnan Province, China. AmJTrop Med Hyg 2010, 81:503-509.

58. McMichael AJ, Martens WJM: The health impact of global climate changes: grasping with scenarios, predictive models and multiple uncertainties. Ecosyst Hlth 1995, 1:23-33.

59. Ree HI: Studies on Anopheles sinensis, the vector species of vivax malaria in Korea. Korean J Parasitol 2005, 43:75-92

60. Lee HI, Lee JS, Shin EH, Lee WJ, Kim YY, Lee KR: Malaria transmission potential by Anopheles sinensis in the Republic of Korea [in Korea] Korean J Parasitol 2001, 39(2):185-192

61. Lindsay SW, Martens WJM: Malaria in the Africa highlands, 1998: past, present and future. Bull World Health Organ 1998, 76:33-45.

62. Zhou GC, Zhang HW, Su YP, Zhou SS, Fang H: Evaluation of therapeutic measures of radical treatment for malaria in pre-transmission season in Yongcheng, Henan, China in 2007. J Pathogen Biol 2009, 4(2):112-114.

doi:10.1186/1471-2458-12-544

Cite this article as: Zhang et al:: Spatial-temporal analysis of malaria and the effect of environmental factors on its incidence in Yongcheng, China, 2006-2010. BMC Public Health 2012 12:544.

\section{Submit your next manuscript to BioMed Central and take full advantage of:}

- Convenient online submission

- Thorough peer review

- No space constraints or color figure charges

- Immediate publication on acceptance

- Inclusion in PubMed, CAS, Scopus and Google Scholar

- Research which is freely available for redistribution 